This item was submitted to Loughborough's Research Repository by the author.

Items in Figshare are protected by copyright, with all rights reserved, unless otherwise indicated.

\title{
Transition state geometry of driven chemical reactions on time-dependent double-well potentials
}

\section{PLEASE CITE THE PUBLISHED VERSION}

http://dx.doi.org/10.1039/C6CP02519F

\section{PUBLISHER}

Royal Society of Chemistry / ( T) The Owner Societies

\section{VERSION}

AM (Accepted Manuscript)

\section{PUBLISHER STATEMENT}

This work is made available according to the conditions of the Creative Commons Attribution-NonCommercialNoDerivatives 4.0 International (CC BY-NC-ND 4.0) licence. Full details of this licence are available at: https://creativecommons.org/licenses/by-nc-nd/4.0/

\section{LICENCE}

CC BY-NC-ND 4.0

\section{REPOSITORY RECORD}

Junginger, Andrej, Galen T. Craven, Thomas Bartsch, F. Revuelta, F. Borondo, R.M. Benito, and Rigoberto Hernandez. 2016. "Transition State Geometry of Driven Chemical Reactions on Time-dependent Double-well Potentials". Loughborough University. https://hdl.handle.net/2134/21926. 


\title{
Transition state geometry of driven chemical reactions on time-dependent double-well potentials
}

\author{
Andrej Junginger, ${ }^{1, a)}$ Galen T. Craven,, , b) Thomas Bartsch, ${ }^{2}$ F. Revuelta, ${ }^{3,4}$ F. Borondo, ${ }^{4,5}$ R. M. Benito, ${ }^{3}$ and \\ Rigoberto Hernandez $\left.{ }^{1}, \mathrm{c}\right)$ \\ 1) Center for Computational Molecular Science and Technology, School of Chemistry and Biochemistry, \\ Georgia Institute of Technology, Atlanta, GA 30332-0400 \\ ${ }^{2)}$ Department of Mathematical Sciences, Loughborough University, Loughborough LE11 3TU, \\ United Kingdom \\ ${ }^{3)}$ Grupo de Sistemas Complejos, Escuela Técnica Superior de Ingeniería Agronómica, Alimentaria y de Biosistemas, \\ Universidad Politécnica de Madrid, Madrid, Spain \\ 4) Instituto de Ciencias Matemáticas (ICMAT), Cantoblanco, 28049 Madrid, Spain \\ ${ }^{5)}$ Departamento de Química, Universidad Autónoma de Madrid, Cantoblanco, 28049 Madrid, \\ Spain
}

Reaction rates across time-dependent barriers are difficult to define and difficult to obtain using standard transition state theory approaches because of the complexity of the geometry of the dividing surface separating reactants and products. Using perturbation theory (PT) or Lagrangian descriptors (LDs), we can obtain the transition state trajectory and the associated recrossing-free dividing surface. With the latter, we are able to determine the exact reactant population decay and the corresponding rates to benchmark the PT and LD approaches. Specifically, accurate rates are obtained from a local description regarding only direct barrier crossings and to those obtained from a stability analysis of the transition state trajectory. We find that these benchmarks agree with the PT and LD approaches for obtaining recrossing-free dividing surfaces. This result holds not only for the local dynamics in the vicinity of the barrier top, but also for the global dynamics of particles that are quenched at the reactant or product wells after their sojourn over the barrier region. The double-well structure of the potential allows for long-time dynamics related to collisions with the outside walls that lead to long-time returns in the low-friction regime. This additional global dynamics introduces slow-decay pathways that do not result from the local transition across the recrossing-free dividing surface associated with the transition state trajectory, but can be addressed if that structure is augmented by the population transfer of the long-time returns.

Keywords: reaction rates, transition state theory

\section{INTRODUCTION}

One of the grand challenges in chemistry is the control of chemical reaction pathways with exquisite specificity. Theoretical and computational approaches require both precise understanding of the potential landscape describing the reactive system and the effect of an external or driving force used to control the reaction. Even with these in hand, the determination of the pathways and rates across these driven chemical processes is challenging. The present work is aimed at addressing this general problem on a prototype chemical reaction through the use of several recent developments in transition state theory (TST) ${ }^{113}$ as discussed below.

Configurational changes of reactive systems are typically mediated by a barrier which separates the reactant and product basins. The detailed dynamics of the reaction is then determined by the structure of the potential

\footnotetext{
a) Present address: Institut für Theoretische Physik, Universität Stuttgart, Germany

b) Present address: Department of Chemistry, University of Pennsylvania, Philadelphia, PA 19104

c) Author to whom correspondence should be addressed; Electronic mail: hernandez@gatech.edu.
}

energy surface (PES) that results from the interactions in the system. For this reason, the PES is of special interest in the investigation of a reaction. On the one hand, it determines the possible reaction pathways and the rate at which a reaction takes place. On the other hand, an incident external driving can lead to modifications of the PES and thereby influence the outcome and speed of the reaction. This is the case, e.g., if tailored external fields are applied that can specifically deform the PES in a desired way 14 An example of such a driven reaction that has recently been addressed using some of the methods described herein is the isomerization of ketene with a time-dependent external field driving its dipole. ${ }^{20}$

In order to describe the rate of a reaction, qualitatively and quantitatively, TST has proven to be an accurate method applicable in many different situations. The fundamental idea of TST is to divide the underlying phase space into regions of reactants and products and to compute the reaction rate using the reactive flux though a dividing surface (DS) separating these regions. The rates calculated using TST are formally exact if the DS is crossed by each reactive trajectory exactly once. Thus, a central task for applying TST is the construction of a DS with this no-recrossings property. In timeindependent systems with a two-dimensional configuration space, the DS is associated with an unstable periodic 
orbit at the barrier top, and in higher-dimensional systems it can be constructed using a normally hyperbolic invariant manifold 2129

A fundamentally different situation exists in the case of systems including time-dependent interactions because the geometric objects used to describe the reaction will then also be time-dependent. The time-dependent transition state (TS) trajectory, ${ }^{30} \sqrt[36]{ }$ which is a distinguished hyperbolic trajectory close to the barrier top, has successfully been applied in several driven systems to obtain formally exact reaction rates in the classical limit. In this context, two observations are of special importance for the practical determination of the reaction rates: First, it has been suggested that for simple barrier structures, 3435 the rate of reactive trajectories crossing the time-dependent DS can be obtained from stability analysis of the TS trajectory. This is a significant simplification because it suggests that the rate may be obtained by investigating only a single trajectory instead of the decay of the reactant population through the laborious propagation of a large ensemble of trajectories. Second, the observation 2013738 that the TS trajectory can be constructed using a minimization procedure of Lagrangian descriptors (LDs) ${ }^{39 / 40}$ has been an important step towards a general construction scheme of this special trajectory on complex energy landscapes.

In previous work, ${ }^{35137}$ some of us addressed a single anharmonic barrier that is externally driven by horizontal oscillations in the barrier position. The agreement between the crossing rates over a barrier with timedependent position and the stability properties of the TS trajectory has thus far been shown for relatively simple potentials described by a barrier and no wells. Including the latter is critical in fully describing chemical reaction dynamics because the bottom of the reactant well is the energetic reference for the temperature of the ensemble of reactants, and it provides the reference frame for the magnitude of the driven oscillations in the barrier. In addition, the existence of a reactant well allows trajectories which leave the barrier region to be reflected at the classical turning point and return to the barrier at longer times. This property, which is particular to the simplified form of the one-dimensional form investigated here at low friction, turns out to be of significant importance for the understanding of the comparison between the overall reaction dynamics observed in numerical simulations and TST calculations.

Thus the major extension in this work involves the use of a model for the time-dependent barrier dynamics which includes reactant and product regions for which reaction rates are well defined using standard approaches. Our results confirm that the TS trajectory provides a recrossing-free DS not only for local trajectories in the vicinity of the barrier region, but also for the entire reactant ensemble. We demonstrate that the nonlocal dynamics in the metastable region does have a significant influence on the total dynamics of the system. The agreement in the results provides further evidence that both
PT and LDs can be used to obtain the TS trajectory. The use of this model introduces long-time dynamics, clearly visible in the numerical trajectories, in which particles return to the barrier after collision on the far side of the bounding potential. These returning trajectories are a consequence of global mechanisms that might be important in chemical reactions but they can nevertheless be accounted for as discussed below.

This paper is organized as follows: In Sec. II, we present the theoretical framework of our investigations by introducing the dynamical equations, model potential energy surface, as well as the theoretical formalism related to the determination of the rate using the TS trajectory, LDs, and stability analysis. In Sec. III] we present numerical results related to the construction of the TS trajectory using LDs, the determination of the reaction rates, and a comparison of the rates with the developed theory. Section IV contains a discussion of the ramifications of our findings and the outlook for future research.

\section{THEORY}

\section{A. The Oscillating Potential}

We now construct a class of time-dependent potentials shown in Fig. 1 which have stationary reactant and product wells but an oscillating anharmonic barrier in the interaction region. Specifically, the potential for this model system is the sum of three terms

$$
V(x, t)=V_{\text {Morse }}^{-}(x)+V_{\text {Gauss }}(x, t)+V_{\text {Morse }}^{+}(x),
$$

where $V_{\text {Morse }}^{ \pm}$are Morse potentials providing wells in the respective reactant $(-)$ and product $(+)$ regions while being small in the region of the other well. A timedependent Gaussian potential $V_{\text {Gauss }}$ provides the dominant structure of the barrier and its motion while behaving approximately harmonically near the barrier top. Specifically, these potential terms are:

$$
\begin{aligned}
V_{\text {Morse }}^{ \pm}(x) & =D\left[1-\mathrm{e}^{ \pm b\left(x \mp x_{0}\right)}\right]^{2}-D, \\
V_{\text {Gauss }}(x, t) & =E_{\mathrm{G}} \mathrm{e}^{-a\left[x-x_{\mathrm{b}}(t)\right]^{2}},
\end{aligned}
$$

where $x_{\mathrm{b}}$ is the position of the barrier, $a$ and $b$ determine the barrier and ground state frequencies, respectively, $E_{\mathrm{G}}$ is the barrier height, $D$ is the well depth, and $\pm x_{0}$ are the positions of the wells. Throughout this paper, we will set $E_{G}=D=a=b=1$, and $x_{0}=3$. This defines, together with $m=1$, a set of natural units for our problem.

To a good approximation the moving Gaussian barrier is zero near the minima of the Morse potentials, and conversely the double well potential formed by the two Morse potentials is constant near the barrier. As a consequence, the total potential has stationary wells at $\pm x_{0}$ and a barrier of constant height and constant shape at 

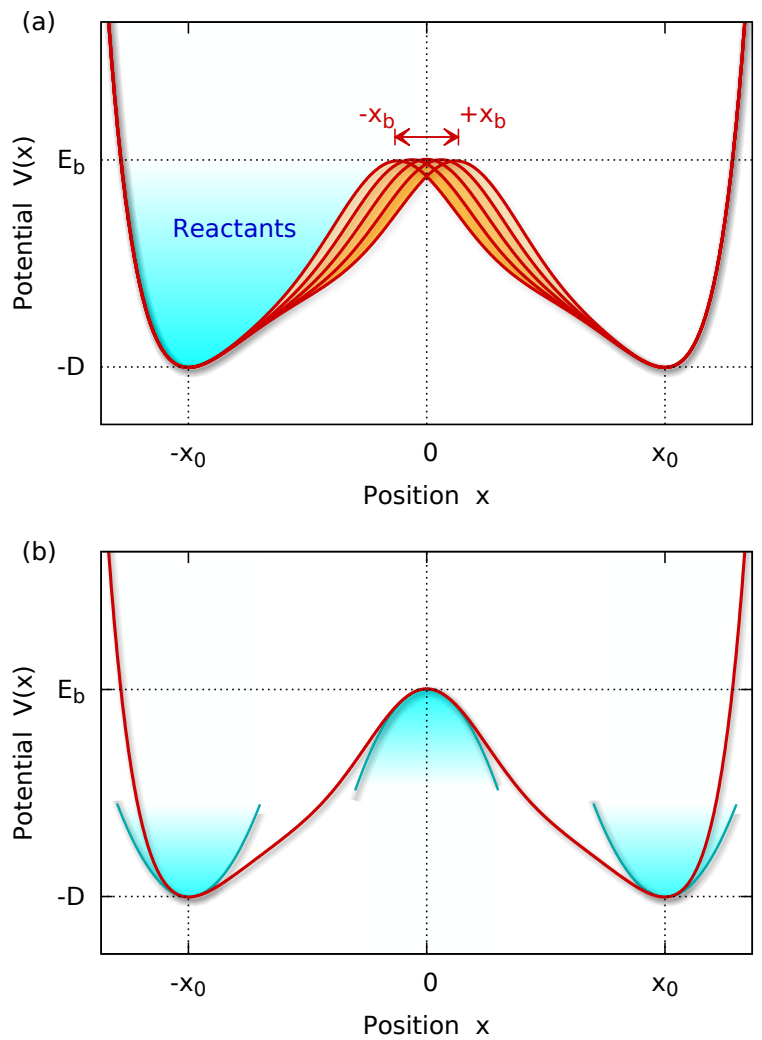

FIG. 1. Spatial dependence of the double-well potential $V(x, t)$ in Eq. (1) for the parameters $E_{\mathrm{G}}=a=D=b=$ $x_{0} / 3=1$. Panel (a) shows the potential for different barrier positions $x_{\mathrm{b}}=0, \pm 0.2 a, \pm 0.4 a$ in red. We consider particles in the left well $x<0$ as reactants. In (b) the harmonic approximations of the potential at its minima and its maximum, respectively, are shown in blue.

$x_{\mathrm{b}}(t)$. In practice, for the parameter values chosen in this work, the barrier height is constant to within $1 \%$ and the location of the maximum differs from $x_{\mathrm{b}}(t)$ by less than $10 \%$.

We use three different protocols for the horizontal oscillation in the position of the barrier top as also employed in Ref. 35:

$$
\begin{aligned}
& x_{\mathrm{b}}^{(1)}(t)=x_{\mathrm{b}}^{\max } \sin \left(\omega_{0} t\right), \\
& x_{\mathrm{b}}^{(2)}(t)=\frac{x_{\mathrm{b}}^{\max }}{2}\left[\cos \left(\omega_{0} t\right)+\cos \left(3 \omega_{0} t\right)\right], \\
& x_{\mathrm{b}}^{(3)}(t)=\frac{x_{\mathrm{b}}^{\max }}{4}\left[\sin \left(2 \omega_{0} t\right)+\sin \left(4 \omega_{0} t\right)+\sin \left(6 \omega_{0} t\right)\right] .
\end{aligned}
$$

The time-dependence for each of these barrier positions is shown in the corresponding top panel of Fig. 2. In this figure, as well as throughout this paper unless stated otherwise, we set $\omega_{0}=\pi$, and $x_{\mathrm{b}}^{\max }=0.4$. The first protocol $x_{\mathrm{b}}^{(1)}(t)$ is a simple single-mode sinusoidal oscillation of the barrier top with frequency $\omega_{0}$ and amplitude $x_{\mathrm{b}}^{\max }$ [see Fig. 2(a)]. The second and third driving protocols also take into account the second and third harmonic of the fundamental frequency [see Figs. 2(b)-(c)] corresponding to the leading-order contributions in a Fourier cosine series.

\section{B. Equation of Motion}

We consider the dynamics of a particle of unit mass in the double-well potential (1), both for the isolated system and for a particle influenced by a thermal bath. In the latter case, the dynamics of the particle is described by the one-dimensional Langevin equation

$$
\ddot{x}(t)=-V^{\prime}(x)-\gamma v(t)+\xi_{\alpha}(t) .
$$

Here, $\gamma$ denotes the strength of the bath-induced friction, $v=\dot{x}$ is the velocity, and $\xi_{\alpha}$ are random forces simulating collisions with solvent particles. The random forces obey the fluctuation-dissipation theorem 141

$$
\left\langle\xi_{\alpha}(t), \xi_{\alpha}\left(t^{\prime}\right)\right\rangle=2 \gamma k_{\mathrm{B}} T \delta\left(t-t^{\prime}\right),
$$

where $k_{\mathrm{B}}$ is the Boltzmann constant and $T$ is the temperature (the index $\alpha$ shall denote a specific random force sequence of the numerical simulation). From this, the description of the isolated system without a bath is obtained for $\gamma=T=0$ and it reduces to the standard Newtonian equations of motion (EoM).

\section{Calculation of the TS Trajectory}

In order to obtain exact rates for trajectories crossing the oscillating barrier (the reactive flux), knowledge of a DS free of recrossings is essential. Otherwise, reactive trajectories that recross the DS lead to overestimates in the flux calculation, and consequently an overestimate of the reaction rate. For several one-dimensional systems with a time-dependent barrier, a recrossing-free DS has been shown to be given by the TS trajectory

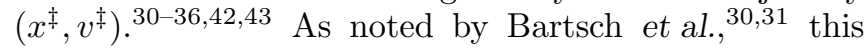
distinguished trajectory defines the origin of a moving coordinate system

$$
\Delta x=x-x^{\ddagger}(t), \quad \Delta v=v-v^{\ddagger}(t),
$$

in which the dynamics (4) effectively becomes timeindependent and noiseless in the harmonic limit. The solution of (4) in this limit is simply given by

$$
\begin{aligned}
& \Delta x(t)=\frac{\mathrm{e}^{\lambda_{\mathrm{u}} t}-\mathrm{e}^{\lambda_{\mathrm{s}} t}}{\lambda_{\mathrm{u}}-\lambda_{\mathrm{s}}}\left[\Delta v(0)-\lambda_{\mathrm{s}} \Delta x(0)\right], \\
& \Delta v(t)=\frac{\lambda_{\mathrm{u}} \mathrm{e}^{\lambda_{\mathrm{u}} t}-\lambda_{\mathrm{s}} \mathrm{e}^{\lambda_{\mathrm{s}} t}}{\lambda_{\mathrm{u}}-\lambda_{\mathrm{s}}}\left[\Delta v(0)-\lambda_{\mathrm{s}} \Delta x(0)\right],
\end{aligned}
$$

where $\lambda_{\mathrm{u}}$ and $\lambda_{\mathrm{s}}$ are the harmonic approximations to the TS trajectory stability exponents, given by

$$
\lambda_{\mathrm{u}, \mathrm{s}}=-\frac{1}{2}\left(-\gamma \pm \sqrt{\gamma^{2}+4 \omega_{\mathrm{b}}^{2}}\right)
$$



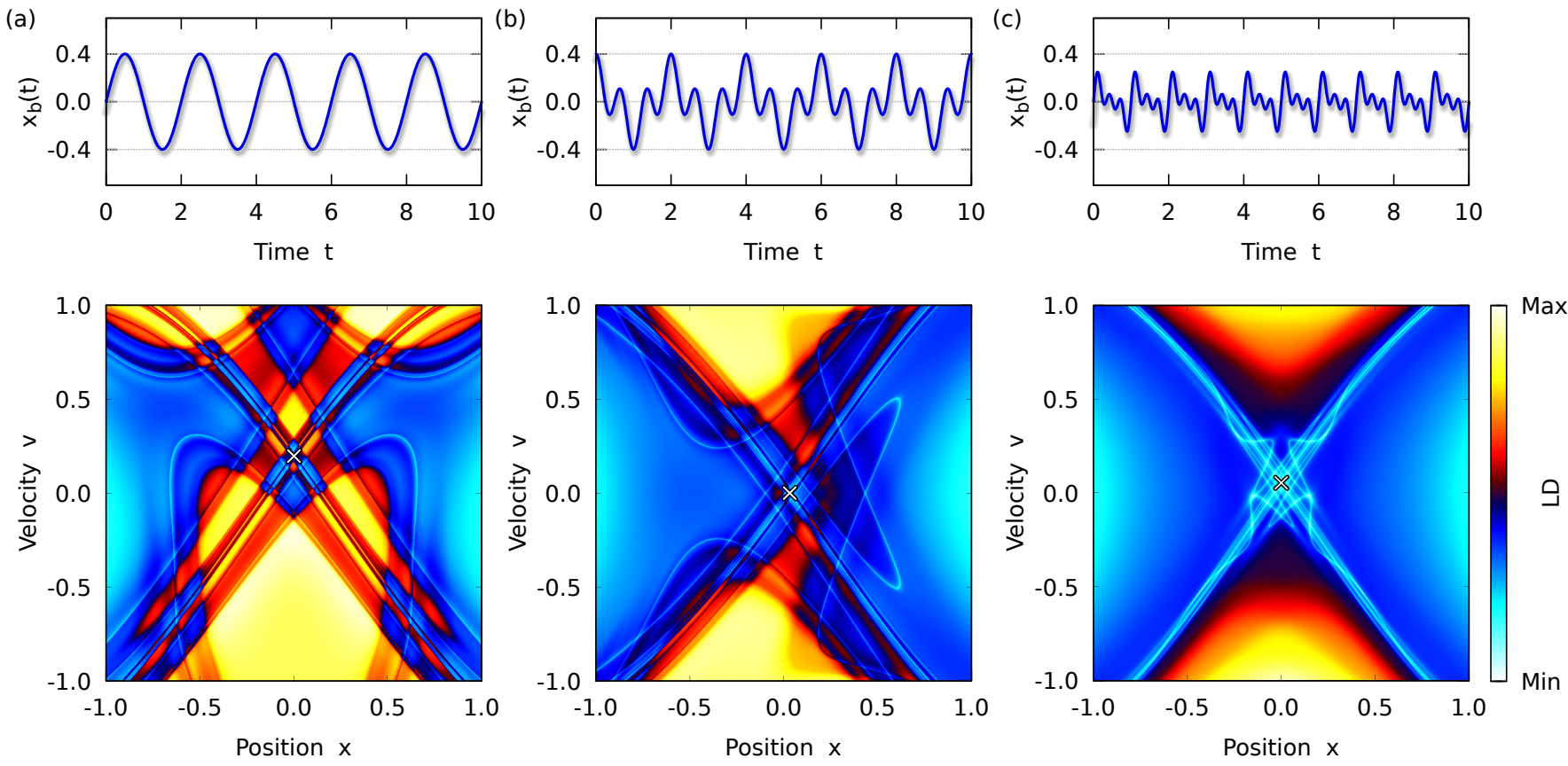

FIG. 2. (Top) Time-dependent barrier position $x_{\mathrm{b}}(t)$ in Eq. (3) for the different driving protocols and parameters $x_{\mathrm{b}}^{\text {max }}=0.4$ and $\omega_{0}=\pi$. (Bottom) Phase space plots of the $\operatorname{LD}(17)$ for $\tau=13, \gamma=0$, and calculated for a grid of size $1000 \times 1000$. The color scale is in arbitrary units and the minimum of the LD is highlighted by the white cross.

and $\omega_{\mathrm{b}}$ is the characteristic barrier frequency. For the Gaussian barrier, its value is $\omega_{\mathrm{b}}=\sqrt{2 a E_{\mathrm{G}}}$. For the total potential in Eq. (1), the barrier frequency is slightly higher than the Gaussian value and is time-dependent. Indeed for the parameter values used in this work, it differs from the Gaussian approximation by less than $5 \%$. For simplicity, we therefore approximate the frequency as if it is constant and equal to that of the Gaussian barrier throughout this work.

For anharmonic barriers, the EoM (4) have no analytic solution. As a consequence, the TS trajectory can only be computed numerically. We report here two alternative procedures for this purpose: First, in Sec. II C 1, we describe a perturbative scheme, and second, in Sec. II C 2, we discuss a more global calculation of the TS trajectory based on LDs.

\section{The TS Trajectory and Perturbation Theory}

Perturbation theory 42,44 (PT) has previously been successfully applied to the determination of the TS tra- jectory, in situations where the potential barrier is not too strongly anharmonic. PT solves the EoM (4) by first making a Taylor expansion of the external potential $V$ as a function of a small parameter, $\varepsilon$, up to a certain order, $n_{\mathrm{PT}}$, and then computing the solution of the EoM order by order. Specifically, the TS trajectory is expanded as

$$
x^{\ddagger}(t)=\sum_{n=0}^{n_{\mathrm{PT}}} \varepsilon^{2 n} x_{n}^{\ddagger}(t),
$$

and setting $\varepsilon=1$ at the end of the calculations. The forces exerted by the moving potential and the noise can then be expanded similarly:

$$
\begin{aligned}
f & =-V^{\prime}\left(x^{\ddagger}(t), t\right)+\xi_{\alpha}(t) \\
& =\sum_{n=0}^{n_{\mathrm{PT}}} \varepsilon^{2 n} f_{n}(t) .
\end{aligned}
$$

For the potential in Eq. (1), the lowest order terms of $f$ are given by 


$$
\begin{gathered}
f_{0}=-\omega_{\mathrm{b}}^{2} x_{\mathrm{b}}+2 b^{2} D \mathrm{e}^{-2 b\left(x_{0}+x_{\mathrm{b}}\right)}\left(x_{\mathrm{b}}-x_{0}^{\ddagger}\right)\left(-\mathrm{e}^{b\left(x_{0}+x_{\mathrm{b}}\right)}-\mathrm{e}^{b\left(x_{0}+3 x_{\mathrm{b}}\right)}+2 \mathrm{e}^{4 b x_{\mathrm{b}}}+2\right)+\xi_{\alpha}(t), \\
\begin{aligned}
f_{1}=a \omega_{\mathrm{b}}^{2}\left(x_{\mathrm{b}}-x_{0}^{\ddagger}\right)^{3}+\frac{1}{3} \mathrm{e}^{-2 b\left(x_{0}+x_{\mathrm{b}}\right)}\left[b^{4} D\left(x_{\mathrm{b}}-x_{0}^{\ddagger}\right)^{3}\left(-\mathrm{e}^{b\left(x_{0}+x_{\mathrm{b}}\right)}-\mathrm{e}^{b\left(x_{0}+3 x_{\mathrm{b}}\right)}+8 e^{4 b x_{\mathrm{b}}}+8\right)\right. \\
\left.-6 b^{2} D x_{0}^{\ddagger}\left(-\mathrm{e}^{b\left(x_{0}+x_{\mathrm{b}}\right)}-\mathrm{e}^{b\left(x_{0}+3 x_{\mathrm{b}}\right)}+2 \mathrm{e}^{4 b x_{\mathrm{b}}}+2\right)\right], \\
f_{2}=-\frac{1}{2} a \omega_{\mathrm{b}}^{2}\left(x_{\mathrm{b}}-x_{0}^{\ddagger}\right)^{2}\left[a\left(x_{\mathrm{b}}-x_{0}^{\ddagger}\right)^{3}+6 x_{1}^{\ddagger}\right] \\
+\frac{1}{60} \mathrm{e}^{-2 b\left(x_{0}+x_{\mathrm{b}}\right)}\left[b^{6} D\left(x_{\mathrm{b}}-x_{0}^{\ddagger}\right)^{5}\left(-e^{b\left(x_{0}+x_{\mathrm{b}}\right)}-e^{b\left(x_{0}+3 x_{\mathrm{b}}\right)}+32 \mathrm{e}^{4 b x_{\mathrm{b}}}+32\right)\right. \\
-60 b^{4} D x_{1}^{\ddagger}\left(x_{\mathrm{b}}-x_{0}^{\ddagger}\right)^{2}\left(-\mathrm{e}^{b\left(x_{0}+x_{\mathrm{b}}\right)}-\mathrm{e}^{b\left(x_{0}+3 x_{\mathrm{b}}\right)}+8 \mathrm{e}^{4 b x_{\mathrm{b}}}+8\right) \\
\left.-120 b^{2} D x_{2}^{\ddagger}\left(-\mathrm{e}^{b\left(x_{0}+x_{\mathrm{b}}\right)}-\mathrm{e}^{b\left(x_{0}+3 x_{\mathrm{b}}\right)}+2 \mathrm{e}^{4 b x_{\mathrm{b}}}+2\right)\right] .
\end{aligned}
\end{gathered}
$$

The perturbative terms of the TS trajectory expansion in Eq. (9) are given by $33 \sqrt[35 / 44]{3}$

$$
x_{n}^{\ddagger}(t)=\frac{1}{\lambda_{\mathrm{u}}-\lambda_{\mathrm{s}}}\left(S\left[\lambda_{\mathrm{u}}, f_{n} ; t\right]-S\left[\lambda_{\mathrm{s}}, f_{n} ; t\right]\right),
$$

where the $S$-functionals ${ }^{32}$ are defined as

$$
S[\lambda, g ; t]= \begin{cases}-\int_{t}^{\infty} g(\tau) \exp (\lambda(t-\tau)) d \tau & : \operatorname{Re} \lambda>0, \\ +\int_{-\infty}^{t} g(\tau) \exp (\lambda(t-\tau)) d \tau & : \operatorname{Re} \lambda<0 .\end{cases}
$$

Because $f_{n}$ depends on the low order terms, $x_{0}^{\ddagger}, x_{1}^{\ddagger}, \ldots, x_{n}^{\ddagger}$, Eq. 12 is an implicit equation for $x_{n}^{\ddagger}(t)$ that can be solved by a fixed point iteration.

Figure 3 shows the TS trajectory computed using the previous perturbative scheme for the three driving protocols introduced in Eq. (3) up to order $n_{\mathrm{PT}}=4$. Here, we have also superimposed the TS trajectory (black dashed curve) computed using the LDs described in the next subsection; it is taken as a reference since it provides a more accurate solution. As can be seen, the general behavior of the TS trajectory is nearly captured by the harmonic approximation to the TS trajectory (leading order term in PT), $x_{0}^{\ddagger}$, as shown in red. However, a more accurate result is obtained when higher orders are included, as can be inferred from the figure. In general, the agreement between LDs and PT increases with the perturbation order, $n_{\mathrm{PT}}$. Nevertheless, for the second driving protocol (3b), the results obtained for $n_{\mathrm{PT}}=1$ (blue) fit those of the LD better than those obtained when $n_{\mathrm{PT}}=2$ (orange), $n_{\mathrm{PT}}=3$ (green), or $n_{\mathrm{PT}}=4$ (yellow).

In order to obtain a numerically exact representation of the TS trajectory that includes all orders of perturbation theory at once, we separate the harmonic part of the barrier potential from the anharmonic contributions and rewrite the equation of motion (4) for the TS trajectory $x^{\ddagger}$ as

with

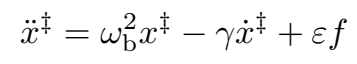

$$
f=-V^{\prime}\left(x^{\ddagger}(t), t\right)-\omega_{\mathrm{b}}^{2} x^{\ddagger}(t)+\xi_{\alpha}(t) .
$$

If $\varepsilon=1$, Eq. (14) agrees with the original equation of motion (4). If $\varepsilon=0$, it reduces to the trivial case of the harmonic barrier without external driving or noise.

Using the methods of Refs. 30, 31, 42, and 43, it can be shown that the solution of Eq. 14 can be formally expressed as

$$
x^{\ddagger}(t)=\frac{\varepsilon}{\lambda_{\mathrm{u}}-\lambda_{\mathrm{s}}}\left(S\left[\lambda_{\mathrm{u}}, f ; t\right]-S\left[\lambda_{\mathrm{s}}, f ; t\right]\right),
$$

Equation $(16)$ is an implicit equation for the unknown function $x^{ \pm}(t)$. It can be solved by a fixed point iteration, starting from the known solution, $x^{\ddagger}=0$ for $\varepsilon=0$. The parameter $\varepsilon$ can be used to control the convergence of the iteration scheme by computing the TS trajectory first for small values of $\varepsilon$ and then increasing $\varepsilon$ towards the limiting value equal to 1 which is the case of interest.

The TS trajectory (solid black) given by Eq. (16) is also shown in Fig. 3. The agreement between this TS trajectory and the exact one given by the LDs is very good, especially for the third driving protocol (3c), where it is seen that it clearly improves the PT results cf. inset in Fig. 3(c)]. For the second driving protocol (3b), the results rendered by both approximations are similar. In this case, the PT (9) agrees better than Eq. (16) with the LDs for positive values of the TS trajectory, and worse for the negative ones, as shown in Fig. 3(b). Finally, for the first driving protocol (3a), the results given by Eq. (9) are always in better agreement with those obtained with Eq. 16. if $n_{\mathrm{PT}} \geq 2$.

\section{The TS Trajectory and Lagrangian Descriptors}

An alternative procedure for the computation of the TS trajectory is given by the global minimization of 

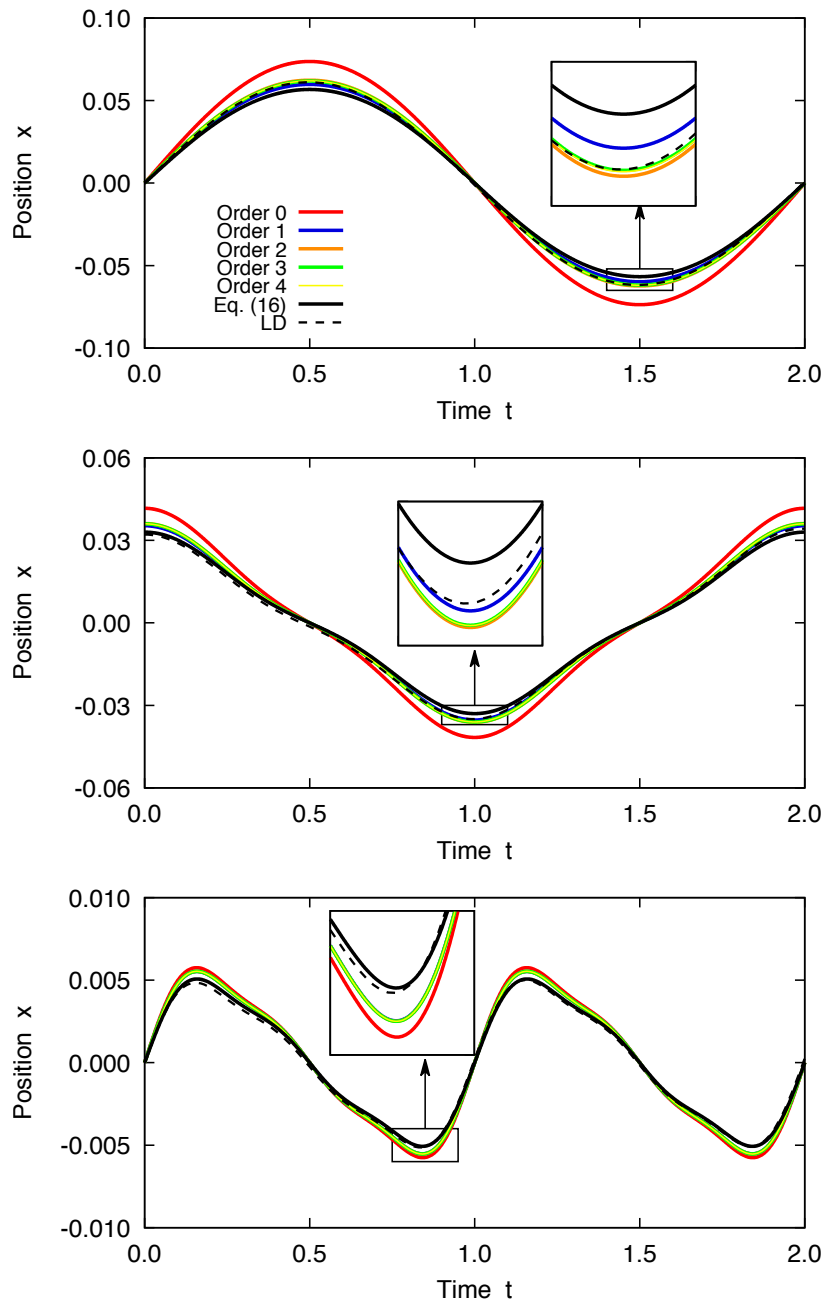

FIG. 3. Time-dependence of the TS trajectory for the driving protocols given by Eqs. (3a), (3b), and $3 \mathrm{c}$ for $\gamma=0$. The black dashed curve shows the results for the LDs, the black solid curve those for Eq. (16), and the other curves the perturbative results obtained with Eq. (9) (leading-order: red; order 1: blue; order 2: orange; order 3: green; order 4: yellow). Notice how the error reduction as the perturbative order is increased. Insets: detailed view of the TS trajectory close to the first minimum.

LDs ${ }^{20137 \sqrt{38}}$ In the context of TST, a particular useful LD is defined by

$$
\mathcal{L}\left(\boldsymbol{x}_{0}, \boldsymbol{v}_{0}, t_{0}\right)=\int_{t_{0}-\tau}^{t_{0}+\tau}\|\boldsymbol{v}(t)\| \mathrm{d} t,
$$

where $\boldsymbol{v}$ is the velocity of a certain trajectory and therefore $\mathcal{L}$ is a measure of arc length of that trajectory over the time interval $\left[t_{0}-\tau ; t_{0}+\tau\right]$. We will also use onesided $\operatorname{LD} \mathcal{L}^{(f)}$ and $\mathcal{L}^{(b)}$ in which the time interval (17) is extended only over the forward $\left(f: t_{0} \leq t \leq t_{0}+\tau\right)$ or the backward $\left(b: t_{0}-\tau \leq t \leq t_{0}\right)$ direction of time.

Of special importance for the reaction dynamics are the stable and unstable manifolds $\mathcal{W}_{\mathrm{s}, \mathrm{u}}$ attached to the (time-dependent) barrier top. These can be identified us- ing the LD in Eq. 17 because the trajectories on these manifolds yield extremal properties of the LD. More precisely, the manifolds directly correspond to minima of the LD in the forward and the backward LD, respectively:

$$
\begin{aligned}
& \mathcal{W}_{\mathrm{s}}\left(\boldsymbol{x}_{0}, t_{0}\right)=\arg \min \mathcal{L}^{(f)}\left(\boldsymbol{x}_{0}, \boldsymbol{v}_{0}, t_{0}\right), \\
& \mathcal{W}_{\mathrm{u}}\left(\boldsymbol{x}_{0}, t_{0}\right)=\arg \min \mathcal{L}^{(b)}\left(\boldsymbol{x}_{0}, \boldsymbol{v}_{0}, t_{0}\right) .
\end{aligned}
$$

The function arg min here denotes the value of the argument $\boldsymbol{v}_{0}$ that yields the minimum of the LD. The coordinates of the TS trajectory $\mathcal{T}\left(t_{0}\right)$ at time $t_{0}$ is located on the intersection $\mathcal{W}_{\mathrm{s}} \cap \mathcal{W}_{\mathrm{u}}$ of the time-dependent stable and unstable manifolds, and it corresponds to the minimum of the two-sided LD (17),

$$
\mathcal{T}\left(t_{0}\right)=\arg \min \mathcal{L}\left(\boldsymbol{x}_{0}, \boldsymbol{v}_{0}, t_{0}\right) .
$$

\section{Stability Analysis}

Motivated by the assumption that the local dynamics of reactive trajectories close to the TS trajectory determines the reaction rate, previous work ${ }^{[34}$ suggests that a linearized stability analysis of this trajectory contains the complete information on the rate. We will compare the corresponding results with the numerical rates of the double-well potential, and therefore present a brief overview on the stability analysis in the following. For details, we refer the reader to Ref. 34 .

The stability analysis hinges on the assumption that the long-time dynamics, which determines the rate, is dominated by the local dynamics close to the TS trajectory, i. e. we consider the case that $\Delta x$ and $\Delta v$ are small enough that the dynamics can be appropriately described by linearized dynamical equations. In this case, a trajectory which is initially close to the TS trajectory evolves in time according to the linear relation

$$
\left(\begin{array}{l}
\Delta x(t) \\
\Delta v(t)
\end{array}\right)=\mathcal{M}(t)\left(\begin{array}{l}
\Delta x_{0} \\
\Delta v_{0}
\end{array}\right)
$$

Here, $\mathcal{M}$ is the stability matrix with the properties

$$
\mathcal{M}(0)=\mathbf{1}, \quad \dot{\mathcal{M}}=\mathcal{J}(t) \mathcal{M},
$$

and $\mathcal{J}$ is the Jacobi matrix of the EoM about the TS trajectory. If the TS trajectory is periodic and characterized by its first single period, $\mathcal{M}$ reduces to the monodromy matrix whose eigenvalues are the Floquet multipliers $m_{\mathrm{s}, \mathrm{u}}$. One of the multipliers, $m_{\mathrm{s}}$, is associated with an approach of the trajectory towards the TS trajectory on the stable manifold, while the other, $m_{\mathrm{u}}$, corresponds to the increasing deviation from the TS trajectory on the unstable manifold. The corresponding time scales are then given by the Floquet exponents

$$
\mu_{\mathrm{s}, \mathrm{u}}=\frac{1}{T} \log \left|m_{\mathrm{s}, \mathrm{u}}\right| .
$$


Recent work by Craven et al ${ }^{34 \sqrt{35}}$ suggests agreement between the barrier crossing rate and the difference of the Floquet exponents 22 ,

$$
k_{\mathrm{floq}}=\mu_{\mathrm{u}}-\mu_{\mathrm{s}} .
$$

Moreover, Revuelta et al $!^{44}$ have shown that the relation in 23) can be generalized to aperiodic and thermally driven systems if the Floquet exponents are replaced by Lyapunov exponents. We will use these relations for comparisons with the numerical results below.

\section{RESULTS}

In this section, we present the results of our investigation on the double-well potential (1) with oscillating barrier position. We first demonstrate the construction of the time-dependent TS trajectory using LDs. Second, we present the capability of the TS trajectory to serve as a recrossing-free DS in both the noisy and noiseless cases. Third, we compare and contrast the rates obtained from the different methods described in the previous section.

\section{A. TS Trajectory and its Local Dynamics}

Contour plots of the LD over the phase spacecalculated using Eq. (17) - for each of the driving protocols (3) in the noiseless case with no a thermal bath are shown in the bottom panels in Fig. 2. According to Eqs. (18), the stable and unstable manifolds $\mathcal{W}_{\mathrm{s}, \mathrm{u}}$ close to the barrier top appear as minimum valleys of the LD. Their intersections are homoclinic points, and the one with the lowest value of the LD naturally corresponds to the TS trajectory [see Eq. (19)]. It is highlighted with a marker ( $x$ in white). Other intersections of the stable and unstable manifolds are also homoclinic points. They are the result of particles that leave the barrier region, that are subsequently reflected by the well, and that finally reenter the barrier region. This is a class of trajectories that did not appear in previous work ${ }^{34}$ because the lack of reactant and product wells in the potentials therein did not give rise to recurrences. It exists only for sufficiently low values of the damping $\gamma$. For large damping, trajectories that leave the barrier region along the unstable manifold are quickly attracted into one of the wells and do not return to the barrier unless reactivated at much longer time scales than those relevant to the rate. The results in Fig. 2 were all obtained for the more intricate case of low damping.

The LD plots in Fig. 2 have an axial symmetry that forces the LD minimum to be located on either the $x=0$ or the $v=0$ axis. This symmetry results from that of the double-well potential (1) with respect to $x=0$ and a suitable choice of the phase of the external driving: The symmetry axis is either the line $x=0$ [in Figs. 2(a), (c)] or the line $v=0$ [in Fig. 2(b)] reveals that the reason is
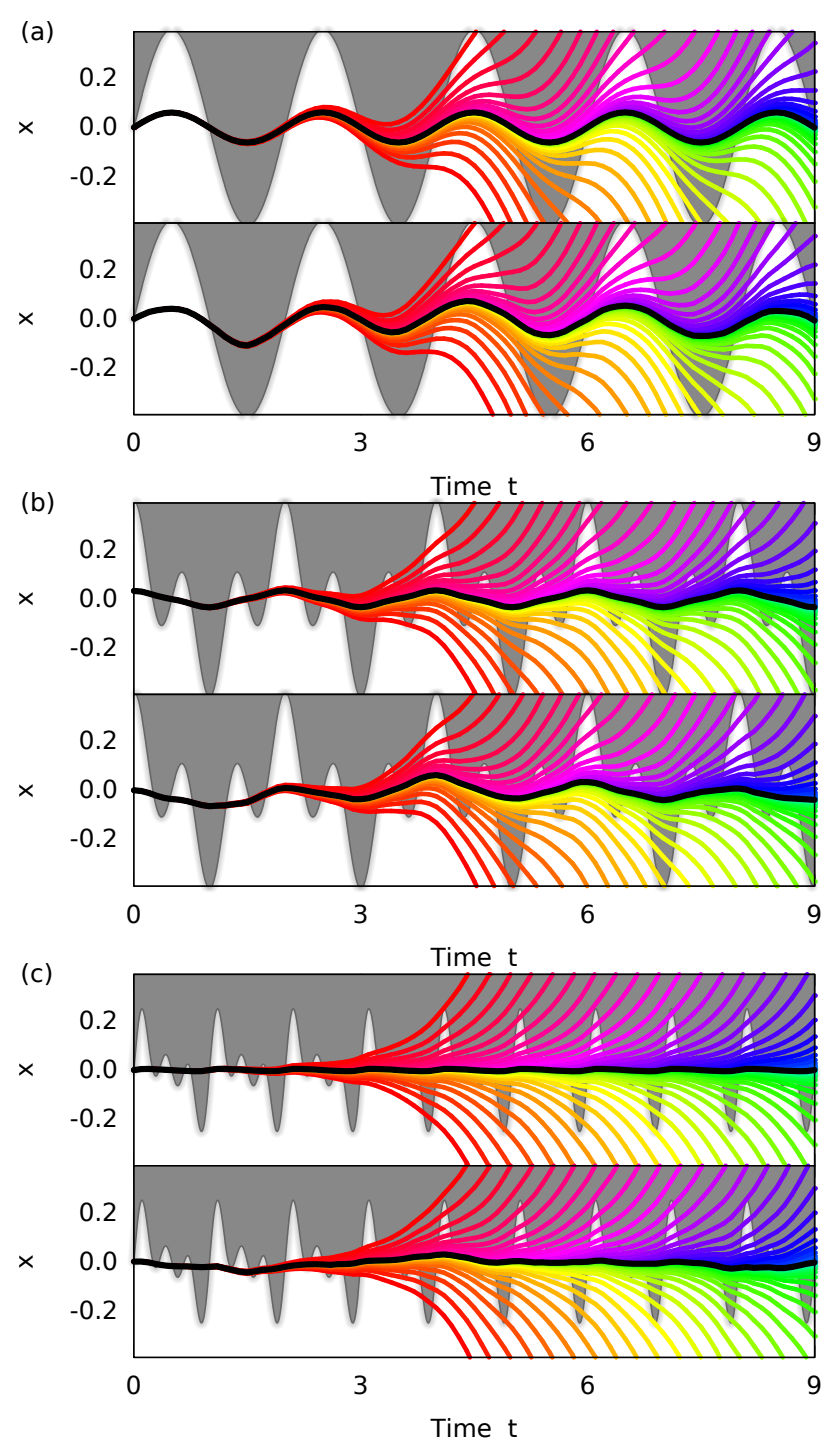

FIG. 4. TS trajectories (solid black) and barrier positions (dotted black) for the three driving protocols in Eqs. (3a-c), respectively. The respective top panels show the dynamics without noise, while the bottom panels include noise and friction with $\gamma=0.001$ and $k_{\mathrm{B}} T=5$.

rather the external driving: the symmetry axis is $x=0$ in case that the external driving is $x_{\mathrm{b}}=0$ at $t=0$ and it is the line $v=0$ if $x_{\mathrm{b}}=x_{\mathrm{b}}^{\max }$ at $t=0$. The symmetry of the LD plots is lost if noisy dynamics with the random forces $\xi_{\alpha}$ in Eq. (4) are taken into account (not shown). If the temperature is not too high, so that the dynamics is still dominated by the underlying potential, noisy dynamics only leads to small changes in the LD structure in phase space. However, the basic structure of the LD, the stable and unstable manifolds, and the existence of the minimum 19 remain. The method of minimizing the LD can, therefore, also be used to extract the TS trajectory in this case.

The TS trajectories for noisy and noiseless dynamics 
for each of the driving protocols 1-3 are shown in Fig. 4 as black curves. Panels (a)-(c) correspond to the respective subfigures of Fig. 2. Within each panel, the top figure shows the case without noise, while the bottom figure includes noise. Furthermore, the white background color denotes the region $x<x_{\mathrm{b}}$ left to the barrier and the gray background is the region $x>x_{\mathrm{b}}$ on its right-hand side. The border between the two regions consequently displays the position of the barrier top $x_{\mathrm{b}}$. The colored curves show the time evolution of representative particles which have been initially placed close to the TS trajectory at $t=0$. As can be seen, the corresponding trajectories escape strictly either to the left or the right of the TS trajectory, crossing it at most once. This observation indicates that the TS trajectory carries a recrossing-free dividing surface, as required by TST.

\section{B. Global Structure and Reaction Dynamics}

The invariant manifolds of the TS trajectory that were obtained by the LD also provide a global description of the dynamics. In particular, the stable manifold of the TS trajectory contains trajectories that are trapped near the barrier top for all time. It therefore separates regions of reactive and non-reactive trajectories in phase space. According to Eq. (18), the location of the stable manifold is indicated by a valley of minima of the backward LD. In addition, the forward-time LD changes drastically as the stable manifold is crossed. For trajectories on the nonreactive side of the stable manifold the velocity decreases to zero as they approach the barrier. The forward LD of these trajectories is therefore markedly smaller than for reactive trajectories, which slow down near the barrier, but do not come to a complete stop. Fig. 5 compares of the forward-time $(0 \leq t \leq \tau)$ LD and the basins of attraction of the left and right wells for various values of the damping $\gamma$. In all cases, the contour lines on the surface computed using LDs (top panels) are in excellent agreement with the corresponding contours of final state committers (bottom panels). For a noiseless system, the TS trajectory is a periodic orbit. Its stable manifold can be calculated in the immediate vicinity of the TS trajectory by a linear stability analysis and further away by backward propagation of trajectories on the manifold. Results of this computation are indicated by the green curves in Fig. 5. They demonstrate both that the stable manifold separates reactive from nonreactive trajectories and that the location of the stable manifold can be reliably obtained from the LD. In noisy systems, where the direct computation of the stable manifold cannot be carried out easily, LD can therefore be applied with confidence.

The reaction rates can be determined from the corresponding construction of the TS trajectory for each of the driving protocols including and excluding noise. For this purpose, we use a thermal ensemble consisting of 10 million particles on the reactant side of the bar- rier with a Boltzmann distribution at a temperature of $k_{\mathrm{B}} T=5.0$. We chose this value of the temperature as it is high enough that a significant amount of particles react, while it is low enough that the dynamics is still dominated by the underlying potential.

The time dependence of the reactant population (top panels) and the number of forward/backward reactions (bottom panels) for each of the driving protocols 1-3 with and without noise are shown in the bottom and top rows of Fig. 6. respectively. In order to define these populations, one has to specify where exactly the reactant well ends. We use either the instantaneous barrier top or the TS trajectory to define the boundary. In the former case, the population shows pronounced oscillations as a function of time that persist well beyond the time interval shown in the figure. By contrast, the TS trajectory gives rise to a monotonic decay of the reactant population, which indicates that it provides a recrossing-free DS.

These observations are underscored by the net number of particles which cross the DS in a given time interval, as indicated in the bottom panels of Fig. 66 (positive values mean forward reactions and negative values mean backward reactions). Using the barrier top as the DS, successive forward and backward reactions occur throughout the entire time interval. Furthermore, one observes a clear correlation of these forward/backward reactions with the motion of the barrier top (the barrier top velocity $v_{\mathrm{b}}$ is shown as a black curve): Forward and backward reactions result when $v_{\mathrm{b}}<0$ and $v_{\mathrm{b}}>0$, respectively.

The situation is fundamentally different when the TS trajectory $x^{\ddagger}$ is used as a DS (orange curves and histograms): The reactant population decay is monotonous throughout for all driving protocols with and without noise. Further evidence for this behavior is seen in the time evolution of the net number of reactions: here only positive values occur because only forward reactions take place. Therefore, the TS trajectory shows its ability to serve as a recrossing-free DS also in the present case of a time-dependent double-well potential. We emphasize that this observation goes beyond being the confirmation of previous findings based on time-dependent barrier without wells. ${ }^{34}$ The double-well potential allows particles to escape from the local barrier region, traverse the reactant well, be reflected and reenter the barrier region. The observation that the escape of particles which have left and reentered the barrier region can be characterized by corresponding TS trajectories that are recrossing-free extends the previous observations from a local to a global picture.

\section{Barrier Crossing Rates}

In addition to characterizing the nature of trajectories as reactive or nonreactive, the TS trajectory can be used to also compute the rates by which particles evolve from the reactant to the product well. For comparison, 

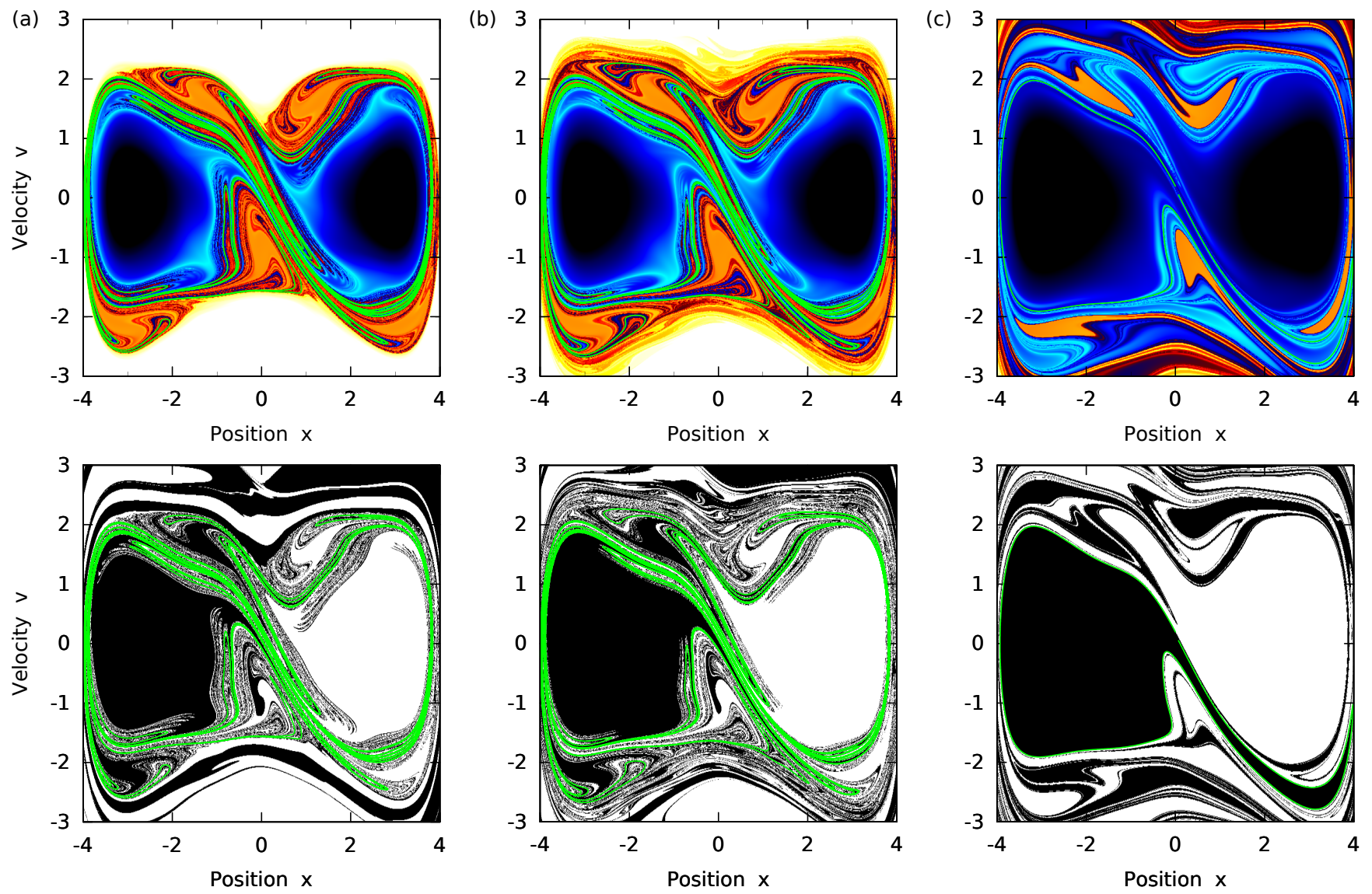

FIG. 5. (Top) Global phase space portrait of the forward contribution of the LD $(0 \leq t \leq \tau)$ for Protocol 1 on the whole range relevant for the reaction dynamics in the double-well potential and different values of the friction: (a) $\gamma=0.00,(b) \gamma=0.01$, and (c) $\gamma=0.05$. The LD has been computed for the parameter $\tau=40$ and the color scale is the same as in Fig. 2 (Bottom) Corresponding plots showing the basins of attraction for a particle initiated at the respective phase space position. Black and white refer to the left and right well as the corresponding basins, respectively.

we obtain these rates $(k)$ numerically by monitoring the population decay and fitting it to the function

$$
\mathcal{P}(t)=\mathcal{P}_{1} \mathrm{e}^{-k t}+\mathcal{P}_{2} t+\mathcal{P}_{3},
$$

where $\mathcal{P}_{1,2,3}$ and $k$ are fitting parameters. Such a form typically includes only two terms, $\mathcal{P}_{1} \mathrm{e}^{-k t}$ and $\mathcal{P}_{3}$, corresponding to the exponential decay towards the "longtime" asymptotic value in the population. However, due to the driven oscillations in the barrier, energy is constantly pumped into the reactant ensemble. Consequently, a mean decrease in the reactant population is observed at long times $(t \gtrsim 10$ for the parametric values employed here) that can be captured by a decreasing linear term. This linear decrease is given by the contribution, $\mathcal{P}_{2} t+\mathcal{P}_{3}$ in Eq. (24), shown as a dotted black curve in the comparisons of the fit to the population decay in Fig. 6 .

Before we discuss the detailed dependence of the reaction rates on different parameters such as temperature, barrier oscillation frequency and amplitude, we first address a comparison between particles crossing the barrier directly and those that first leave the barrier region and traverse the reactant well before a reaction takes place. For this purpose, we consider an initial thermal ensemble $\rho_{\delta}$ with a $\delta$-distribution at $x^{\prime}$ in configuration space and a Boltzmann distribution $\rho_{v}(v)$ in velocity space,

$$
\rho_{\delta_{x^{\prime}}}(x, v) \propto \delta\left(x-x^{\prime}\right) \times \rho_{v}(v) .
$$

The family of such ensembles allows for a more detailed analysis of the dynamics, but it does not prevent the determination of the average decay of the population because the entire thermal ensemble $\rho_{\text {therm }}$ can be recovered simply through integration over the complete reactant well,

$$
\rho_{\text {therm }}(x, v)=\int_{\text {well }} \mathrm{d} x^{\prime} \rho_{\delta_{x^{\prime}}}(x, v) .
$$

Figure 7(a) shows the time development of the reactant population for the initial distribution 25 with $x^{\prime}=-0.2$, i. e. close to the barrier top. The decay of the reactant population does not decrease through a single near-exponential decay but rather exhibits a series of stepped decays. The first step is related to those trajectories (not shown) which have enough kinetic energy as 

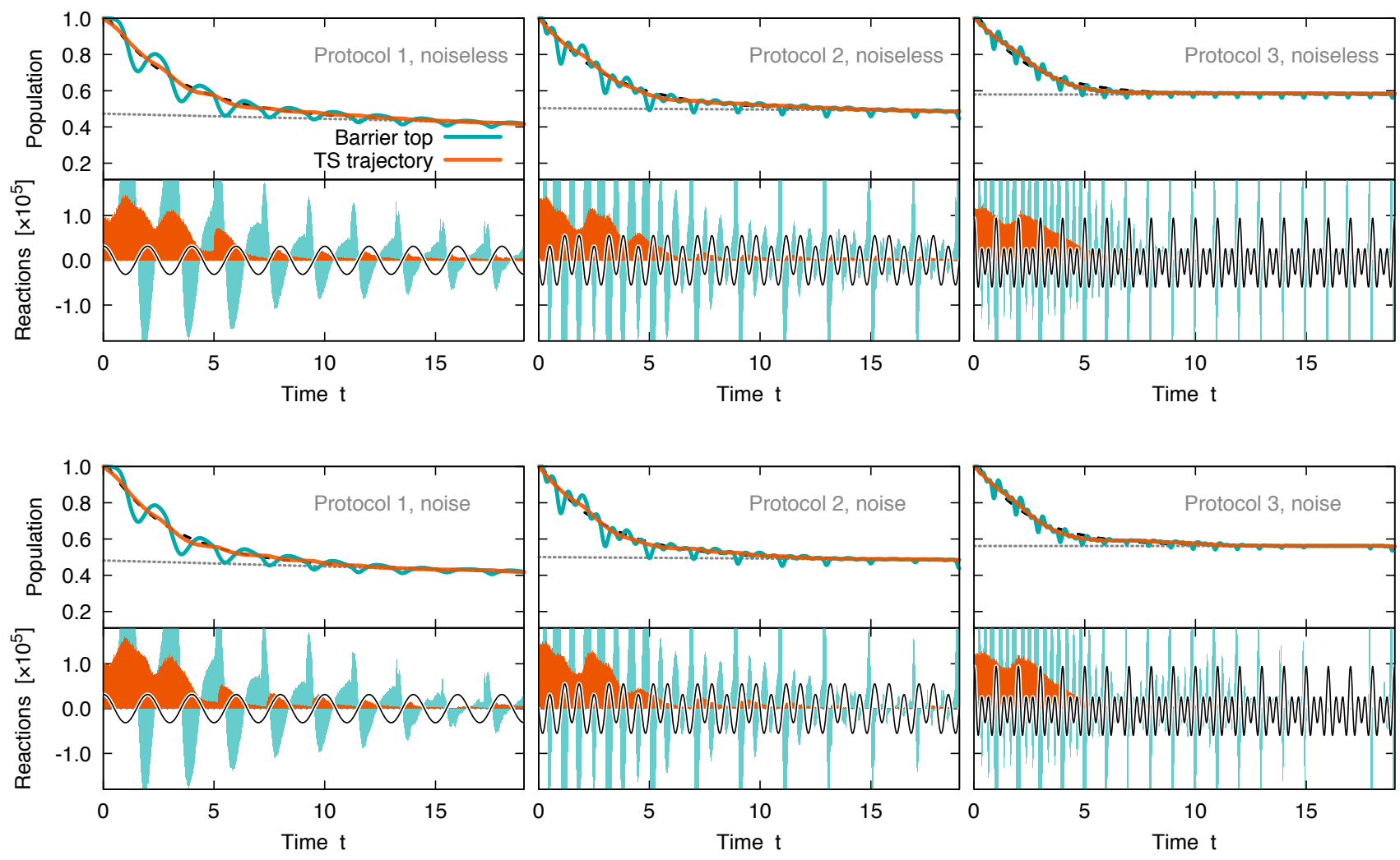

FIG. 6. Comparison of the reaction dynamics at a temperature of $k_{\mathrm{B}} T=5$ obtained from the barrier top as DS (cyan) and the TS trajectory as DS (orange). The respective top panels show the time-dependence of the reactant population $\mathcal{P}(t)$. The bottom panels present histograms showing the number reacting particles per time interval $\Delta t=0.01$. Positive values indicate forward reactions, while negative numbers mean backward reactions. The solid black curve indicates the barrier velocity (in arbitrary units) for each driving protocol. The black dashed curves show the long-time decay contribution $\mathcal{P}_{2} x+\mathcal{P}_{3}$ in Eq. 24 . In the noisy case, the calculations have been performed with a friction parameter $\gamma=0.001$

well as a positive value of the velocity $v>0$ near the barrier such they cross it directly, leading to products. By contrast, the second step is due to particles which first leave the barrier region into the reactant well, are reflected by the back wall, return to the barrier, and then react. Further steps correspond to two, three, and more oscillations in the reactant well before the reaction takes place. This suggests that the overall rate $k_{\text {tot }}$ is a convolution of the direct decay - as characterized by the direct rate $k_{\text {dir }}$ - within a given step and the population remaining after each of the many possible long-time recurrences due to multiple reflections with the back wall.

As displayed in Fig.7(a), both the direct and total decay mechanisms lead to different values of the rate. The direct rate $k_{\text {dir }}$ is significantly larger: an exponential fit to the first drop-off of the reactant population in Fig. 7 (black dashed curve) yields a rate of $k_{\text {dir }}=3.491$ while the overall fit (taking into account the several-step reaction; black dash-dotted curve) corresponds to a rate of $k_{\text {tot }}=0.184$. In Fig. 77(b), we present the same procedure for the several-step decay for different positions $x^{\prime}$ of the initial distribution $\rho_{\delta_{x^{\prime}}}$. It shows that the initial position $x^{\prime}$ has a significant influence on the locations and the sizes of the single drop-offs. The reason for this hinges predominantly on whether a given particle in the ensemble has enough initial kinetic energy to cross the barrier. The overall reaction rate of the total thermal ensemble in the reactant well is obtained from the average (26). It is shown as the red solid curve in Fig. 7 and the corresponding rate can be extracted from the fit shown as the black dash-dotted curve.

An alternative interpretation of the difference in the direct and total rates can also be inferred following the observations of De Leon et al ${ }^{[45 / 46}$ about the role of reactive islands on rates. The geometry of the interfaces between the basins of attraction in Fig. 5 in the vicinity of the barrier is characterized by several distinct but parallel components of the DS. The direct rate results from the crossing of the first of these in the barrier region whereas the total rate includes trajectories that cross (in the reactive direction) any one of them. Meanwhile, the loss of exponential behavior that we find in some of the cases below would presumably result from the chaotic structure in the DSs between the reactive islands. 

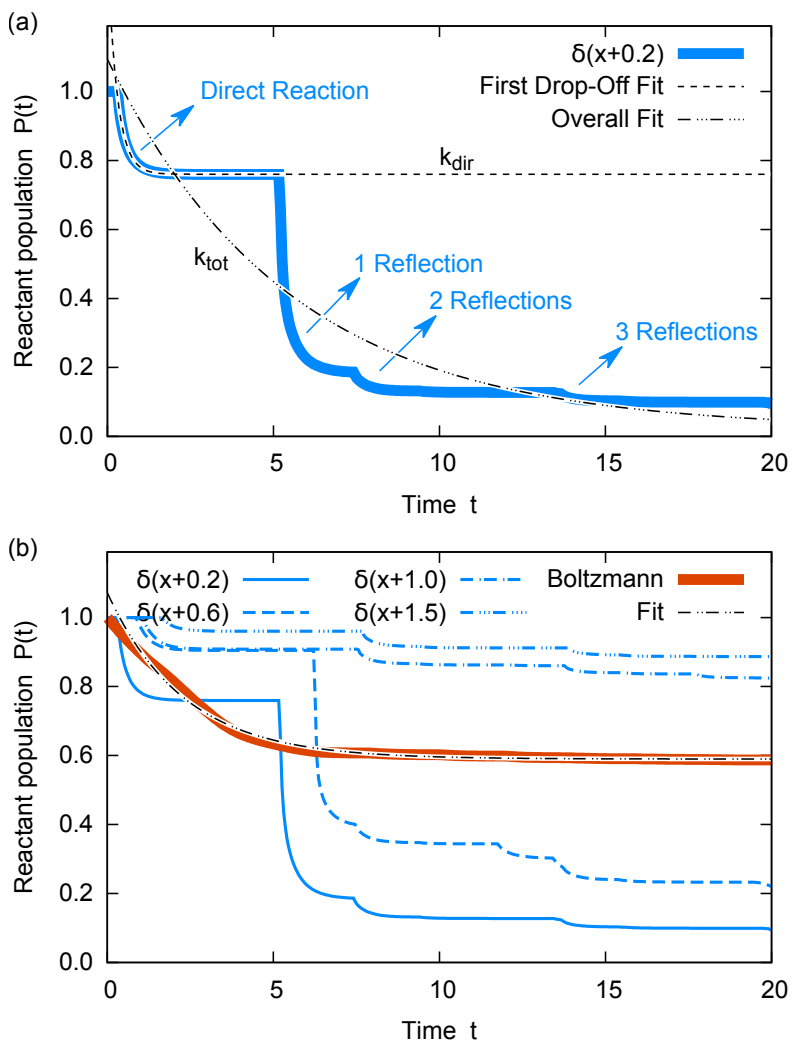

FIG. 7. (a) Time-dependence of the reactant population for an initial $\delta$-distribution at $x=-0.2$. The dashed curve shows an exponential fit 24 with rate $k_{\text {floq }}$ to the first drop-off of the population $(0 \leq t \leq 5)$. The dash-dotted curve shows the same exponential fit with decay rate $k_{\text {tot }}$ to the whole time scale $(0 \leq t \leq 20)$. Panel (b) demonstrates the different population decay in dependence of the position of the initial $\delta$-distribution in blue. The corresponding behavior for a Boltzmann distribution which results from integrating over all possible $\delta$-distributions is shown in red.

\section{Direct vs Global Rates}

Reaction rates as a function of temperature $k_{\mathrm{B}} T$ [Fig. 8(a)], barrier frequency $\omega_{0}$ [Fig. 8(b)], and barrier amplitude $x_{\mathrm{b}}^{\max }$ [Fig. 8(c)] are shown in Fig. 8, We also show the rates obtained from three different methods: (i) the rates $k_{\text {tot }}$ (dots connected with dashed lines) are obtained from the decay of a thermal ensemble distributed over the whole reactant well, (ii) the rates $k_{\text {dir }}$ (dots without lines) obtained from an ensemble which has an initial distribution (25), and (iii) the rates $k_{\text {floq }}$ (solid curves) obtained from the Floquet difference 23) which provide a good approximation to the TS trajectory stability exponents under small noise strength.

In the temperature dependence of the rates, one naturally expects an exponential behavior according to

$$
k=\text { const } \times \mathrm{e}^{-\Delta E / k_{\mathrm{B}} T} .
$$

This expectation is indeed satisfied for the overall rates
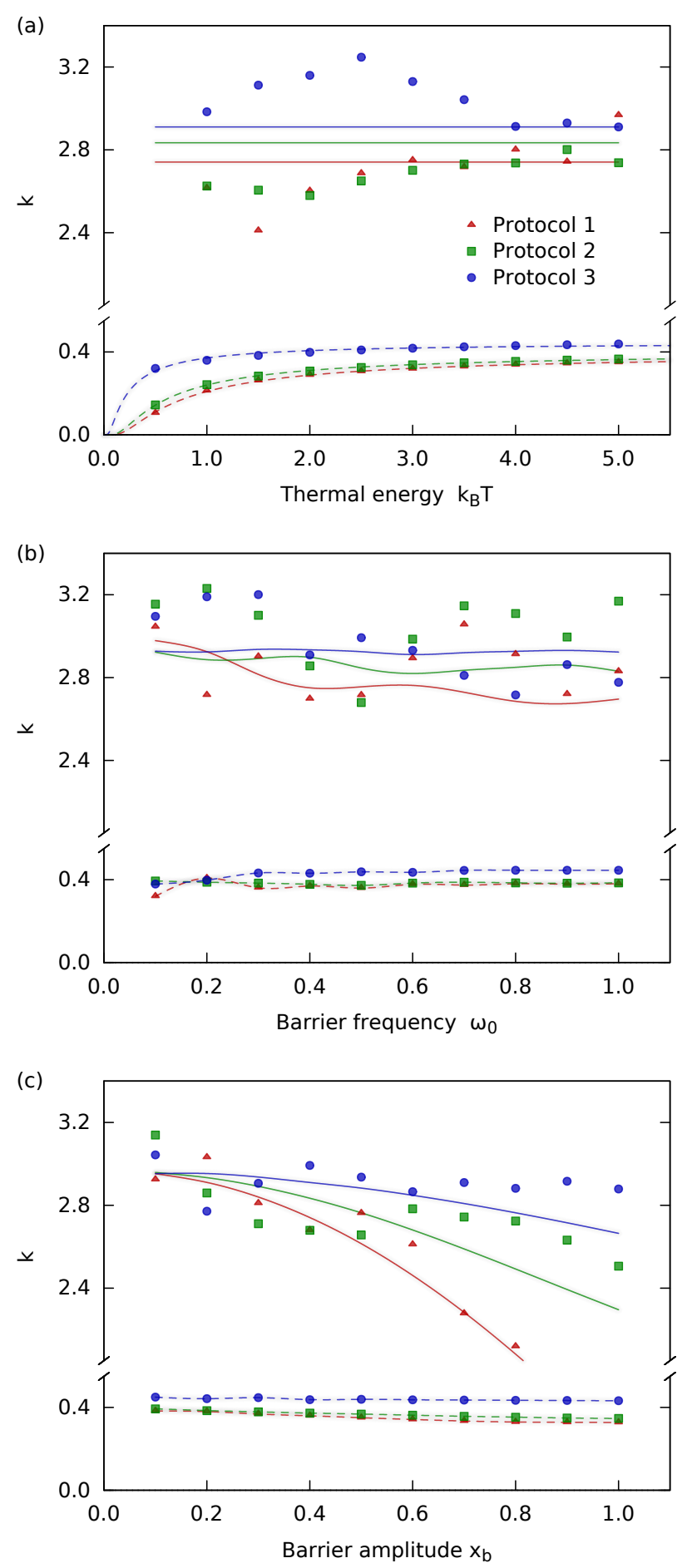

FIG. 8. Comparison of the rates $k_{\text {floq }}$ obtained from the difference of the Floquet exponents (solid lines), the rates $k_{\text {tot }}$ observed from the decay of the reactant population (dots connected with dashed lines), and the rates $k_{\text {dir }}$ of the direct reaction (dots without lines). Shown is the dependence on (a) the temperature $k_{\mathrm{B}} T$ of the initial ensemble, (b) the barrier frequency $\omega_{0}$, and (c) the barrier amplitude $x_{\mathrm{b}}$. The dots show the rates obtained from a fit to the reactant population decay according to Eq. 24). The lines in (a) show fits of Eq. 27) to the data points (see Tab. I for the fit coefficients), and the lines in $(b, c)$ are cubic interpolations of the data. 


\begin{tabular}{ccl}
\hline Protocol & const & $\Delta E$ \\
\hline 1 & 0.3972 & 0.6422 \\
2 & 0.4026 & 0.5169 \\
3 & 0.4456 & 0.1834 \\
\hline
\end{tabular}

TABLE I. Fit coefficients of Eq. (27) to the data shown in Fig. 8(a).

$k_{\text {tot }}$ in Fig. 8(a) with the corresponding fits to (27) shown as dotted curves. The fit coefficients are provided in Tab. I. Although the exponential behavior is satisfied, the rates differ by up to one order of magnitude. In contrast to these cases, the Floquet rate $k_{\text {floq }}$ in Eq. (23) does not reproduce the total rates. This disagreement results from the fact that the Floquet rate is obtained only from the stability properties of the TS trajectory, leaving out the possibility for longer decays due to the reflections. The TS trajectory is independent of the initial distribution and therefore does not depend on the corresponding temperature. Therefore, this is also true for the Floquet rates, and Floquet rates appear as lines of constant height in Fig. 8(a).

In Fig. 8(b), we see that a different behavior of the rates also manifests itself in its dependence on the barrier frequency $\omega_{0}$. While the total rates $k_{\text {tot }}$ remain the smallest throughout, the direct rates $k_{\text {dir }}$ are, again, the highest ones, and the Floquet rates lie in between. All the rates, however, have in common that a slight modulation is observed when varying the frequency [for the driving protocol 2 and at low frequency (green) it is even a large modulation].

As we demonstrate in Fig. 8(c), the general behavior of the direct crossing rates $k_{\text {dir }}$ and the Floquet rate $k_{\text {floq }}$ is similar if the barrier amplitude is varied. In both cases, the rate changes only marginally for small amplitudes, while it decreases faster as the barrier amplitude becomes larger. The dependence of the direct and Floquet rates seen here also agree in the sense that the single-mode driving (red) shows the strongest decrease, while it is weakest for protocol 3 . By contrast, the total rate $k_{\text {tot }}$ does not show such a decrease in the rate when increasing the oscillation amplitude of the barrier. Because the total rate $k_{\text {tot }}$ is the only one that takes into account the nonlocal structure of the potential apart from the barrier, we expect that this effect is due to the fact that the total rate is dominated by the structure of the well and influenced by the barrier motion to a minor degree.

A general observation in Figs. 8(b)-(c) is the fact that the barrier driving amplitude and frequency are less relevant for the total crossing rates $k_{\text {tot }}$ than they are for the direct rates $k_{\mathrm{dir}}$. This observation can, again, be explained by the multi-step decay process shown in Fig. 7 where two different time scales are of importance: The first one is the characteristic time scale of the direct process, which only depends on the local barrier properties, and thus it is sensitive to the barrier driving. The second time scale is the oscillation period of the particle in the well. It is only slightly modified by the driving because of the construction of the potential (1). In other systems in which the driving also has a significant influence on the form of the well (e.g. its depth), a strong dependence of the driving parameters on the total rates may also be observed.

Our rate calculations demonstrate the importance of taking into account the complete dynamics of the system when the global dynamics lead to recurrences to the barrier region. The fact that the direct rates are captured well by the recrossing-free DS associated with the TS trajectory indicates its accuracy. When trajectories can revisit the barrier region due to the presence of reflecting boundaries, one must evidently include this additional mechanism to obtain the long time rates.

\section{CONCLUSIONS}

In this paper, we have investigated the reaction dynamics of a double-well potential with a time-dependent (driven) barrier in a thermal bath using the Langevin equation to describe the dynamics. We have computed the TS trajectory - which serves as a recrossing-free DS using different orders in PT and using a method based on the minimization of LDs. The results of these independent methods are in good agreement up to small variances that would be expected from the different orders of PT.

Through this work, we have verified the recrossingfree property of the TS trajectory by direct integration of thermal ensembles of particles. The results show that this DS is free of recrossings in cases without and with noise, and this property also holds for particles which react after one or more oscillations in the reactant well. The latter result is especially important because it extends previous findings regarding a local picture that is only in the vicinity of the barrier top. What emerges is a global picture that takes into account the whole reactive system.

We have also computed the reaction rates of the system for different temperatures, oscillating frequencies and amplitudes of the driven barrier. The comparison of the rates obtained from the total population decay, the direct barrier crossings, and the Floquet rates underscore the importance of taking into account the complete dynamics of the system: All the comparisons agree with the observation that the total reaction rate is significantly lower than the direct or Floquet rates. The total rates exhibit a monotonic increase with temperature (as would be expected from standard statistical rate theories), the direct rates appear to be convolved with an additional resonance, and the Floquet-derived rates are temperature independent. By contrast, the direct rates and the ones obtained from the Floquet exponents of the TS trajectory agree in their behavior with increasing barrier oscillation amplitude; the total rates do not show such a dependence. 
In summary, this work demonstrates that: (i) The direct rates can be obtained accurately using several methods for determining the TS trajectory. In particular the LD approach leads to accurate rates. (ii) The values of the LDs give rise to a structure that is similar to that of the long-time reactant and product committers, while the underlying stable and unstable Lagrangian manifolds can be seen from the local minima on the LD hypersurface. (iii) When there are recurrences such as in the special case of the double well, the rates become convolved with this additional mechanism, but the contribution of the rates due to the direct reaction across the barrier can still be captured by the rate associated with the TS trajectory.

Real molecular potentials typically differ from the model potential investigated in this paper in two ways: First, their reactant and product wells usually have a different depth, i.e. the activation barriers in forward and backwards direction differ. Second, the potential energy surfaces of most chemical reactions exhibit more than one nontrivial degree of freedom so that the dynamics of the system perpendicular to the reaction coordinate needs to be considered. Our future investigations will take these important steps into account in order to verify the present results also for the case of nonsymmetric potentials and to make the application of the TS trajectory method possible for real molecular reactions driven by external electromagnetic fields.

\section{ACKNOWLEDGMENTS}

This work has been partly supported by the Air Force Office of Scientific Research through Grant No. FA955012-1-0483, and by the Spanish Ministry of Economy and Competitiveness (MINECO) under Contracts No. MTM2012-39101 and MTM2015-63914-P, and by ICMAT Severo Ochoa under Contract SEV-2015-0554. A. J. acknowledges the Alexander von Humboldt Foundation, Germany, for support through a Feodor Lynen Fellowship. Travel between partners was partially supported through the People Programme (Marie Curie Actions) of the European Union's Seventh Framework Programme FP7/2007-2013/ under REA Grant Agreement No. 294974.

\section{REFERENCES}

\footnotetext{
${ }^{1}$ K. S. Pitzer, F. T. Smith, and H. Eyring, The Transition State, Special Publ. (Chemical Society, London, 1962).

${ }^{2}$ P. Pechukas, Annu. Rev. Phys. Chem. 32, 159 (1981), doi:10.1146/annurev.pc.32.100181.001111.

${ }^{3}$ B. C. Garrett and D. G. Truhlar, J. Phys. Chem. 83, 1052 (1979).

${ }^{4}$ D. G. Truhlar, A. D. Issacson, and B. C. Garrett, Theory of Chemical Reaction Dynamics (CRC Press, Boca Raton, FL, 1985), vol. 4, pp. 65-137.
}

${ }^{5}$ G. A. Natanson, B. C. Garrett, T. N. Truong, T. Joseph, and D. G. Truhlar, J. Chem. Phys. 94, 7875 (1991), doi:10.1063/1.460123.

${ }^{6}$ D. G. Truhlar, B. C. Garrett, and S. J. Klippenstein, J. Phys. Chem. 100, 12771 (1996).

${ }^{7}$ D. G. Truhlar and B. C. Garrett, J. Phys. Chem. B 104, 1069 (2000), doi:10.1021/jp9924301.

${ }^{8}$ T. Komatsuzaki and R. S. Berry, Proc. Natl. Acad. Sci. U.S.A. 98, 7666 (2001), doi:10.1073/pnas.131627698.

${ }^{9}$ H. Waalkens, R. Schubert, and S. Wiggins, Nonlinearity 21, R1 (2008), doi:10.1088/0951-7715/21/1/R01.

${ }^{10}$ T. Bartsch, J. M. Moix, R. Hernandez, S. Kawai, and T. Uzer, Adv. Chem. Phys. 140, 191 (2008), doi:10.1002/9780470371572.ch4.

${ }^{11} \mathrm{~S}$. Kawai and T. Komatsuzaki, Phys. Rev. Lett. 105, 048304 (2010), doi:10.1103/PhysRevLett.105.048304.

${ }^{12}$ R. Hernandez, T. Bartsch, and T. Uzer, Chem. Phys. 370, 270 (2010), doi:10.1016/j.chemphys.2010.01.016.

${ }^{13}$ O. Sharia and G. Henkelman, New J. Phys. 18, 013023 (2016), doi:10.1088/1367-2630/18/1/013023.

${ }^{14}$ K. Yamanouchi, $\quad$ Science 295, $1659 \quad$ (2002), doi:10.1126/science.1068449.

${ }^{15}$ B. J. Sussman, D. Townsend, M. Y. Ivanov, and A. Stolow, Science 314, 278 (2006), doi:10.1126/science.1132289.

${ }^{16}$ S. Kawai and T. Komatsuzaki, J. Chem. Phys. 134, 024317 (2011), doi:10.1063/1.3528937.

${ }^{17}$ A. Sethi and S. Keshavamurthy, Phys. Rev. A 79, 033416 (2009), doi:10.1103/PhysRevA.79.033416.

${ }^{18}$ S. Patra and S. Keshavamurthy, Chem. Phys. Lett. 634, 1 (2015), doi:10.1016/j.cplett.2015.05.011.

${ }^{19}$ F. Revuelta, R. Chacón, and F. Borondo, Europhys. Lett. 110, 40007 (2015), doi:10.1209/0295-5075/110/40007.

${ }^{20}$ G. T. Craven and R. Hernandez, Phys. Chem. Chem. Phys. 18, 4008 (2016), doi:10.1039/c5cp06624g.

${ }^{21}$ E. Pollak and P. Pechukas, J. Chem. Phys. 69, 1218 (1978), doi:10.1063/1.436658.

${ }^{22}$ P. Pechukas and E. Pollak, J. Chem. Phys. 71, 2062 (1979), doi:10.1063/1.438575.

${ }^{23}$ R. Hernandez and W. H. Miller, Chem. Phys. Lett. 214, 129 (1993), doi:10.1016/0009-2614(93)90071-8.

${ }^{24}$ R. Hernandez, J. Chem. Phys. 101, 9534 (1994), doi:10.1063/1.467985.

${ }^{25}$ T. Uzer, C. Jaffé, J. Palacián, P. Yanguas, and S. Wiggins, Nonlinearity 15, 957 (2002), doi:10.1088/0951-7715/15/4/301.

${ }^{26} \mathrm{H}$. Teramoto, M. Toda, and T. Komatsuzaki, Phys. Rev. Lett. 106, 054101(1) (2011), doi:10.1103/PhysRevLett.106.054101.

${ }^{27}$ C.-B. Li, A. Shoujiguchi, M. Toda, and T. Komatsuzaki, Phys. Rev. Lett. 97, 028302(1) (2006), doi:10.1103/PhysRevLett.97.028302.

${ }^{28}$ H. Waalkens and S. Wiggins, J. Phys. A 37, L435 (2004), doi:10.1088/0305-4470/37/35/L02.

${ }^{29}$ U. Çiftçi and H. Waalkens, Phys. Rev. Lett. 110, 233201(1) (2013), 10.1103/PhysRevLett.110.233201.

${ }^{30}$ T. Bartsch, T. Uzer, and R. Hernandez, J. Chem. Phys. 123, 204102(1) (2005), doi:10.1063/1.2109827.

${ }^{31}$ T. Bartsch, R. Hernandez, and T. Uzer, Phys. Rev. Lett. 95, 058301(1) (2005).

${ }^{32}$ T. Bartsch, T. Uzer, J. M. Moix, and R. Hernandez, J. Chem. Phys. 124, 244310(1) (2006), cond-mat/0604640.

${ }^{33}$ G. T. Craven, T. Bartsch, and R. Hernandez, Phys. Rev. E 89, 040801(1) (2014).

${ }^{34}$ G. T. Craven, T. Bartsch, and R. Hernandez, J. Chem. Phys. 141, 041106(1) (2014), doi:10.1063/1.489147.

${ }^{35}$ G. T. Craven, T. Bartsch, and R. Hernandez, J. Chem. Phys. 142, 074108(1) (2015), doi:10.1063/1.4907590.

${ }^{36}$ S. Kawai and T. Komatsuzaki, J. Chem. Phys. 131, 224505(1) (2009).

${ }^{37}$ G. T. Craven and R. Hernandez, Phys. Rev. Lett. 115, 148301 (2015), doi:10.1103/PhysRevLett.115.148301. 
${ }^{38}$ A. Junginger and R. Hernandez, J. Phys. Chem. B 120, 1720 (2016), doi:10.1021/acs.jpcb.5b09003.

${ }^{39}$ C. Mendoza and A. M. Mancho, Phys. Rev. Lett. 105, 038501 (2010), doi:10.1103/PhysRevLett.105.038501.

${ }^{40}$ A. M. Mancho, S. Wiggins, J. Curbelo, and C. Mendoza, Commun. Nonlinear Sci. Numer. Simul. 18, 3530 (2013), doi:10.1016/j.cnsns.2013.05.002.

${ }^{41}$ R. Kubo, Rep. Prog. Phys. 29, 255 (1966), doi:10.1088/0034$4885 / 29 / 1 / 306$
${ }^{42}$ F. Revuelta, T. Bartsch, R. M. Benito, and F. Borondo, J. Chem. Phys. 136, 091102 (2012), doi:10.1063/1.3692182.

${ }^{43}$ T. Bartsch, F. Revuelta, R. M. Benito, and F. Borondo, J. Chem. Phys. 136, 224510 (2012), doi:10.1063/1.4726125.

${ }^{44}$ F. Revuelta, G. T. Craven, T. Bartsch, F. Borondo, R. M. Benito, and R. Hernandez, "Theory for transition state structure and reaction rates in solvated systems with energetic anharmonicity," in preparation.

${ }^{45}$ C. C. Marston and N. D. Leon, J. Chem. Phys. 91, 3392 (1989), doi:10.1063/1.456914.

${ }^{46}$ N. D. Leon, J. Chem. Phys. 96, 285 (1992), do:10.1063/1.462516. 